\title{
A Vitruvius Inspired Criterion for the Construction of Polygons
}

\author{
Leno Liberato Mascia ${ }^{1}$
}

Published online: 15 July 2015

(C) Kim Williams Books, Turin 2015

\begin{abstract}
A geometric analysis carried out on three homonymous works, known as the Vitruvian man, by Leonardo da Vinci, Giacomo Andrea da Ferrara and Gulielmo Philandro has identified the equilateral triangle as a common feature, differing only with respect to the central position of the apex. Considerations regarding the intended purpose of the tangential outer arc in the Ferrara's sketch and the possible implications of the two secants, drawn from the mid-point at the base to its intersections with the sides of the square, have provided the basis for a general construction of polygons. The underlying criterion relies on the parameters that define the geometric components of the arc to establish the position of the vertices of the polygon. The fundamental nature of the construction was confirmed through a comparison with an existing procedure by Fletcher, named "squaring the circle".
\end{abstract}

Keywords Vitruvian Man · Giacomo Andrea da Ferrara - Guglielmo Philandro · Leonardo da Vinci · Vitruvian Man · Geometrical constructions · Polygons

\section{Introduction}

The Vitruvian Man is a topic of great interest in Renaissance history for the interpretation of the canon of human proportions described in The Ten Books on Architecture by Roman architect Marcus Vitruvius Pollio (ca. 25 BC), which is illustrated in the form of images of a man fitting into a square and also in a circle (Fernández 2005). In this study I examine the geometric features of three versions of the Vitruvian Man with the view to determine the geometric implications of the

Leno Liberato Mascia

L.Mascia@lboro.ac.uk

1 Loughborough University, Loughborough LE11 3TU, UK 
related drawings in relation to the construction of polygons. This idea stems from reported observations that the extremities of the fingers and the toes of the man in the circle in Leonardo's drawing locate four vertices of an inscribed heptagon (Helbing 2005; Murtinho 2015).

In examining the details of the sketch conserved in Ferrara, nominally attributed to Giacomo Andrea da Ferrara, one notes that the two chords formed by connecting the mid-point between the feet of the man with the two intersections of the arc at the sides of the square, bisect the circumscribing circle at the points corresponding the two lower vertices of an equilateral triangle with its apex at the navel. This is the constitutive triangle for a hexagon inscribed in the circle (Fig. 1a).

A related examination of this iconic expression of human proportions by various Renaissance artists has identified a similar feature in the sketches by Guglielmo Philandro (Fig. 1b), which also alludes to the possible inscription of a polygon within a circle. Accordingly, the two chords obtained by joining the mid-point at the bottom of the outer circle so as to produce tangential contacts with the inner circle, also correspond to the vertices of a constitutive equilateral triangle for the inscription of the hexagon. Since the two works were produced independently, the intrinsic commonalities can only be attributed to fundamental geometric considerations by the two artists in determining the details of the related sketches. It is possible that Philandro determined the radius of the outer circle from measurements of the intersection of the arc with the sides of the square in the Ferrara's drawing. Measurements by the author on a downloaded version of the Ferrara drawing (Reynolds 2012) (http://www.dailymail.co.uk/news/article-2094647/Leonardo-daVincis-Vitruvian-Man-copied-Giacomo-Andrea-da-Ferrara.html\#ixzz2rUxGCOW2) have revealed that the arc is centred at the top of the head, slightly off-set to the right due to possible distortions incurred in reproductions from the original manuscript.

Further, direct measurements made on the Ferrara sketch of the Vitruvian man indicate that the ratio (side of the square) $\div$ (radius of the circle) $\sim 1.70$ (i.e., $\sim \sqrt{ } 3$ ), which corresponds to the ratio diagonal $\div$ side for a regular hexagon. (Note that there are variations in the recorded measurements due primarily to the distortion of the square, where one angle was found to be $87^{\circ}$ ). It can therefore be deduced that the outline of the sketch has evolved from a geometrical basis.

A similar analysis of the geometric features of Leonardo's drawing has revealed that the two tangents to the circle, drawn from the mid-point of the measuring scale, form a $25^{\circ}$ angle on the right side of the drawing and $27^{\circ}$ on the left side. The two points of contact with the circle coincide with the vertices of an isosceles triangle with a $52^{\circ}$ apex at the navel of the man. It is clear that this geometric feature does not contradict Leonardo's statement regarding the equilateral triangle formed by the opening of the legs (Richter 1970: I, 182). The latter is related to the contour lines of the crotch with its apex at the pubic bone, as indicated in Fig. 2a. This present analysis found that bisecting the circle with a compass opening equal to the distance between the tangential points (Fig. 2a) produces with reasonable accuracy the vertices of an inscribed regular heptagon. The maximum lateral deviation from a regular heptagon is $-2.59 \%$ (shorter) with respect to the two sides forming the vertex at the top. The deviation of the base and the other sides is $+1.04 \%$ (larger). 
a

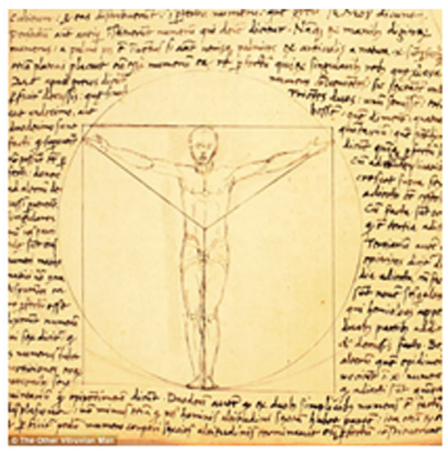

b
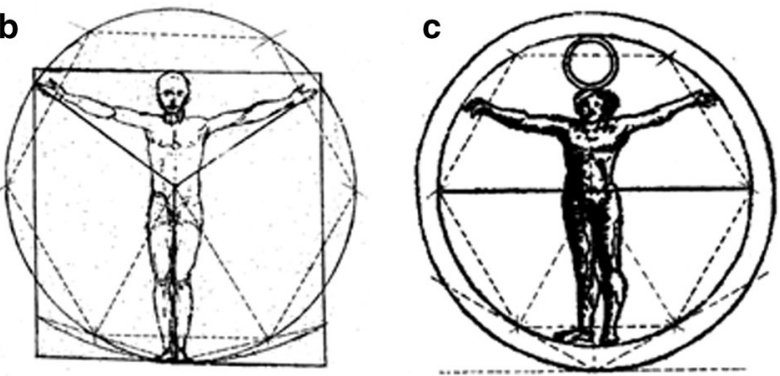

Fig. 1 (Top) a Image of the Vitruvian Man, nominally attributed to Giacomo Andrea da Ferrara. The archivist at the Biblioteca Ariostea in Ferrara confirms that the registration of this sketch is "Vitruvius Pollio Architectura, ms. cart., (1490-1518), Classe II 176". The authenticity of the Ferrara sketch, however, has not yet been proved and the autography is attributed to Pellegrino Prisciani. (I thank Kim Williams for this information). (Bottom) Hexagon inscribed in the circle circumscribing the Vitruvian man (overlaid by the author). b Sketch by Giacomo Andrea da Ferrara. c Sketch by Gulielmo Philandro

The connection of Leonardo's drawing with a heptagonal inscription was recognised by the Renaissance artist Agrippa (March 1998) as well as modern-day scholars (Helbing 2005). This can be inferred directly from rudimental measurements of the distance between the fingers of the man in the circle and the mid-point on the circle at the top, which is approximately equal to the distance between the inner part of the feet. It is instructive to note that the sketches of the man ad quadratum and the man ad circulum in Agrippa's Vitruvian man bare a very close resemblance to Leonardo's drawing as two separate images. However, Agrippa introduces distinct geometric annotations in his drawings, one depicting the square with an inner inverted equilateral triangle (third diagram down in Fig. 2b) and the other delineating the heptagonal inscription in the circle. It can also be deduced that the addition of an equilateral triangle, sharing the base with the heptagon (drawing at the bottom in Fig. 2b), may have been intended by Agrippa to point out the dual significance of Leonardo's drawing. One is concerned with Vitruvius canon regarding the opening of the legs and the related reduction in height of the man, while the other is to make a mathematical statement through the geometric details of the drawing. 

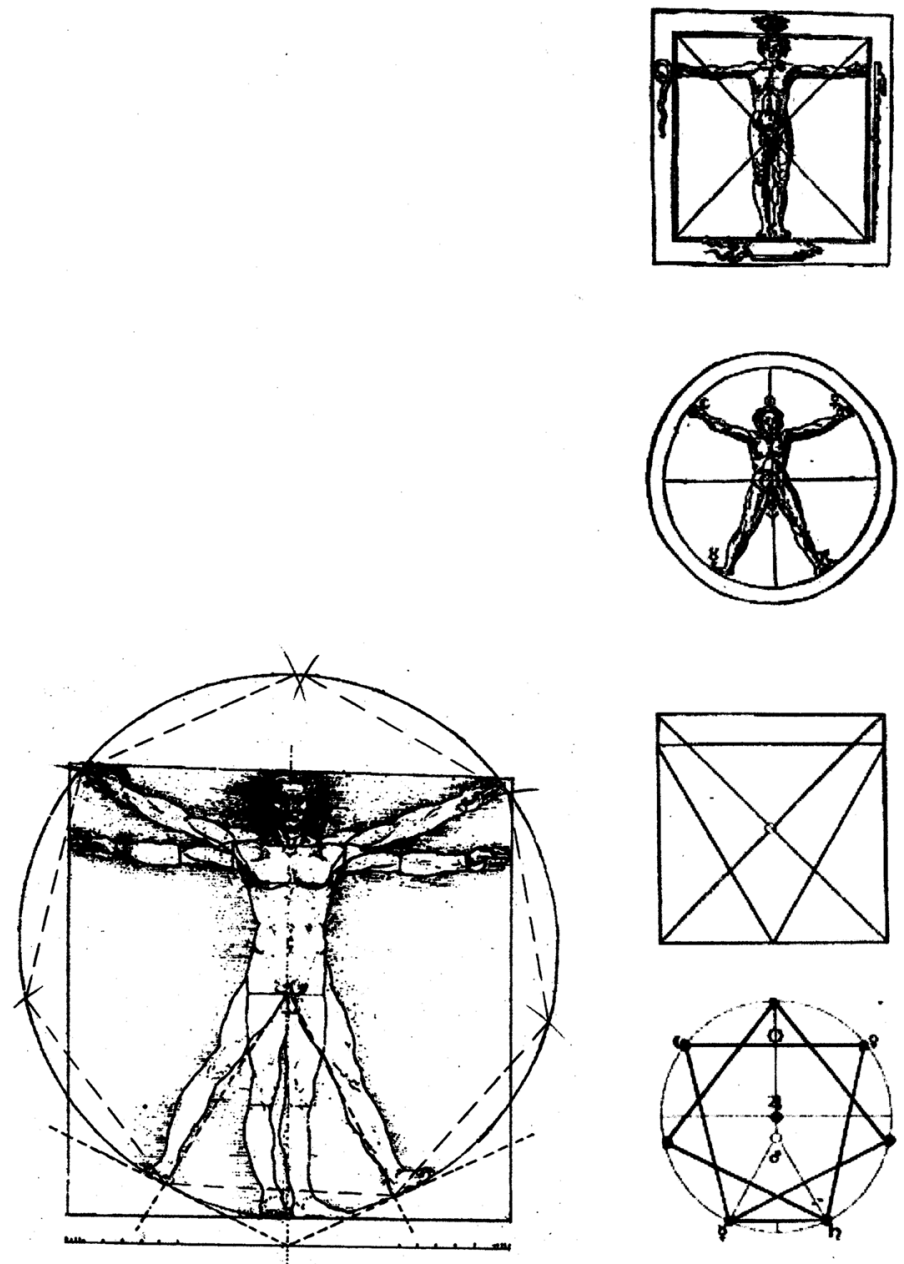

Fig. 2 Two examples of the association of the Vitruvian man with the heptagon: (a, left) Leonardo's depiction of the Vitruvian man, with the heptagon overlaid by the author; (b, right) studies of the human figure by Heinrich Cornelius Agrippa (2003)

A possible association of Leonardo's Vitruvian man with a multi-side polygon can also be inferred from the geometrical details found in an assumed copy of a drawing about human motions by Carlo Urbino (dated c. 1560). It is claimed that this drawing may have originated from Leonardo's lost notes inherent to the Codex Huygens (Lester 2012: 215), in which are included many inscribed polygons, comprising several equilateral triangles, a pentagon, a square and a hexagon within two tangential circles. The diameter of the outer circle corresponds to the full length of the man with his arms raised and joined by the touching of the fingers, while the 
diameter of the smaller circle corresponds to the actual height of the man inscribed within the square. However, there is no allusion of a direct connection of this drawing with Leonardo's Vitruvian man, other than an assertion that Urbino may have been inspired by Leonardo's studies on the relationship between the microcosm and earth (Zöllner 1985).

\section{Arc-Construction of the Heptagon}

In order to explore further the implications of the arc in the sketch attributed to Ferrara for the construction of polygons, I have added this feature to Leonardo's drawing, as shown in Fig. 3a. The centre of the arc was located at the mid-point of the top of the head of the man, while the radius was taken to be equal to the height. This has resulted in an irregular hexagon characterized by a top side that is $24.7 \%$ shorter and five other sides that are are $5.1 \%$ longer than the value for a regular hexagon, allowing for drawing and image reproduction errors. For the diagrams in Fig. $3 b$, on the other hand, the radius of the arc was set to be equal to the diameter of the circle and centred at the mid-point at the top of the circle, allowing a $1^{\circ}$ offset to take into account the gradient of the base, in concordance with the $2^{\circ}$ difference in the inclination angle of the soles of the feet between the two sides of the diagram. The two secants drawn from the central point at the base and intercepting the sides of the square was found to bisect the circle at the points corresponding to the lower vertices of the inscribed polygon.

The resulting heptagon shown in Fig. $3 b$ displays a lateral discrepancy from a regular heptagon in the region of $2 \%$, allowing for drawing errors (see below). It is intriguing to observe that the interception of the cord secant with the inner circles takes place at the same points to those identified by the procedure shown in Fig. 2a.
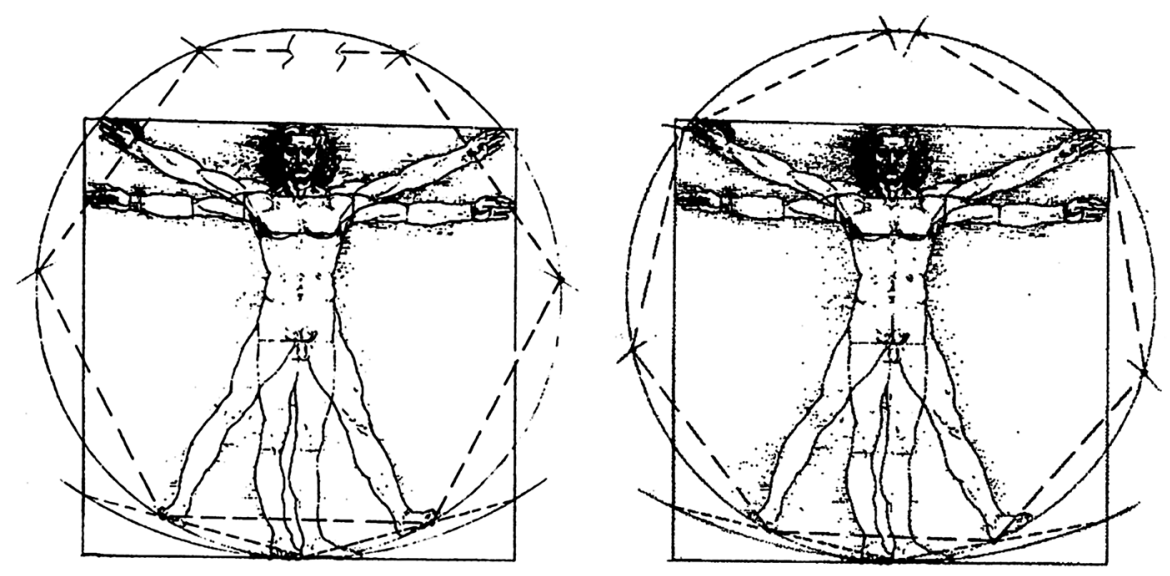

Fig. 3 Construction of polygons in Leonardo's Vitruvian man using the Ferrara arc criterion: (a, left) hexagon derived for arc radius equal to the height of the man; (b, right) heptagon derived for arc radius equal to the diameter of the circle 
How Leonardo managed to set the measuring scale below the square at such an exact distance has not, hitherto, been discussed by scholars.

Through these observations I have realized that a tangential arc and the intersections of the chords from the mid-point at the base to the sides of a rectangle may constitute a general criterion for the construction of polygons and that a relationship between these parameters may exist to derive a procedure for specific polygons. In order to determine the accuracy of the newly identified construction of polygons a more detailed analysis was subsequently carried out using the Autocad software to construct a series of polygons based on the tangential arc criterion. In the first instance the radius of the arc in the Ferrara drawing was reduced from $\Psi \sim \sqrt{ } 3 \mathrm{R}$ to $\Psi=\phi \mathrm{R}$ (where $\phi=1.618$ ), using an arc length $\mathrm{L}=2 \mathrm{R}$ (i.e., the length of the chord corresponding to the width of the perpendicular rectangle with its base tangential to the circle, as shown in Fig. 4a). In doing so the two secants
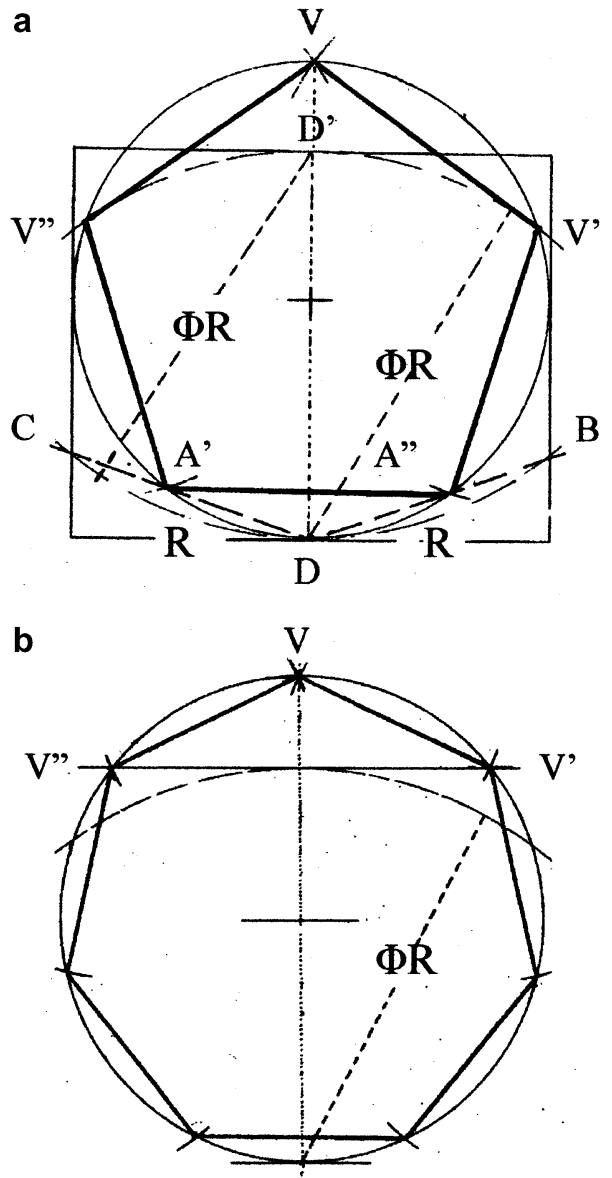

Fig. 4 (a, above) $2 \mathrm{R}$-Arc construction of the pentagon, using parameters $\Psi=\phi \mathrm{R}$ and $\mathrm{L}=2 \mathrm{R}$. (b, below) Construction of the heptagon, using parameters internal arc radius $\rho=\phi \mathrm{R}$ 
drawn from the midpoint at the base (point $\mathrm{D}$ ), intersecting the two sides of the rectangle at $\mathrm{B}$ and $\mathrm{C}$, were found to bisect the circle at points $\mathrm{A}$ and $\mathrm{A}^{\prime}$ and a connecting segment $\mathrm{AA}^{\prime}$ forming the basis of a pentagon in the circumscribing circle. The related construction was completed by drawing an arc of same size, centred at the midpoint on the bottom base of the rectangle (point D), thereby bisecting the circle to form the two top vertices of the pentagon, $\mathrm{V}^{\prime}$ and $\mathrm{V}^{\prime \prime}$. By this procedure the pentagon can be constructed from one single opening of the compass (or, as Leonardo would have said, ... con un solo aprire del seste). However, he may have placed the method into the category falso, since the resulting pentagon has a rather low level of accuracy, exhibiting deviations from the corresponding regular (equilateral) equal to $+5.02 \%$ with respect to the base $\left(\mathrm{AA}^{\prime}\right)$ and $-2.33 \%$ for the two sides $\mathrm{A}^{\prime} \mathrm{V}^{\prime \prime}$ and $\mathrm{A}^{\prime \prime} \mathrm{V}^{\prime}$.

The top two sides $\mathrm{VV}^{\prime}$ and $\mathrm{VV}^{\prime \prime}$, on the other hand, are much closer to that of a regular pentagon, exhibiting a deviation equal to $-0.23 \%$. The association of this procedure with golden ratio may be rooted in the Euclidean construction of the pentagon, which evolves from a horizontal line divided in two segments with lengths proportional to the golden ratio (Pedoe 1976). This has drawn my attention to the possibility of adopting the same procedure to construct a heptagon, since other procedures have also made use of the golden ratio as a parameter (Reynolds 2001; Fletcher 2007). The results of this construction was not expected to be widely different from those emanating from the construction in Fig. 3b, insofar as the ratio $\mathrm{L} / \mathrm{R}$ in Leonardo's drawing is estimated to be in the range 1.64-1.65, as compared with the golden ratio value of 1.618. An internal arc with radius $\Psi=\phi \mathrm{R}$ was used to locate the horizontal line that bisects the circle to form the upper vertices of a heptagon with sides $\mathrm{VV}^{\prime}$ and $\mathrm{VV}^{\prime}$. This heptagon has a maximum deviation from a regular polygon at base, amounting to $-4.38 \%$ (shorter), while for the sides the discrepancy is $+0.75 \%$. From the derived data it can be concluded that the two procedures that locate the vertices of the polygon through an internal arc are highly inaccurate.

\section{The 2R-Arc Construction}

The procedure for the 2R-Arc construction of the heptagon derived from the outlines of Leonardo's Vitruvian Man in Fig. 3b) was re-examined using Autocad software. The construction is illustrated in Fig. 5a using the sides of the square as $\mathrm{L}=\phi \mathrm{R}$ (where $\phi=1.618$ ) to determine the length (L) of the chord formed by the arc, which locates the bottom vertices of the resulting heptagon. The deviation of the length of the two upper sides of the resulting heptagon from that of the corresponding a regular (equilateral) polygon is $-5.77 \%$, which is considerably higher than the $\sim 2 \%$ value estimated for the manual construction derived directly from Leonardo's drawing, based on a nominal golden ratio equal to 1.64. In this respect it should be noted that the exact construction would require a coefficient of $\mathrm{R}$ (i.e., an "equivalent" golden ratio) equal to 1.7355 , which is much higher than the true golden ratio. Using the height of the cord as a parameter, an exact solution would be obtained with a coefficient of $\mathrm{R}$ equal to 1.8019 . Since neither of these two 

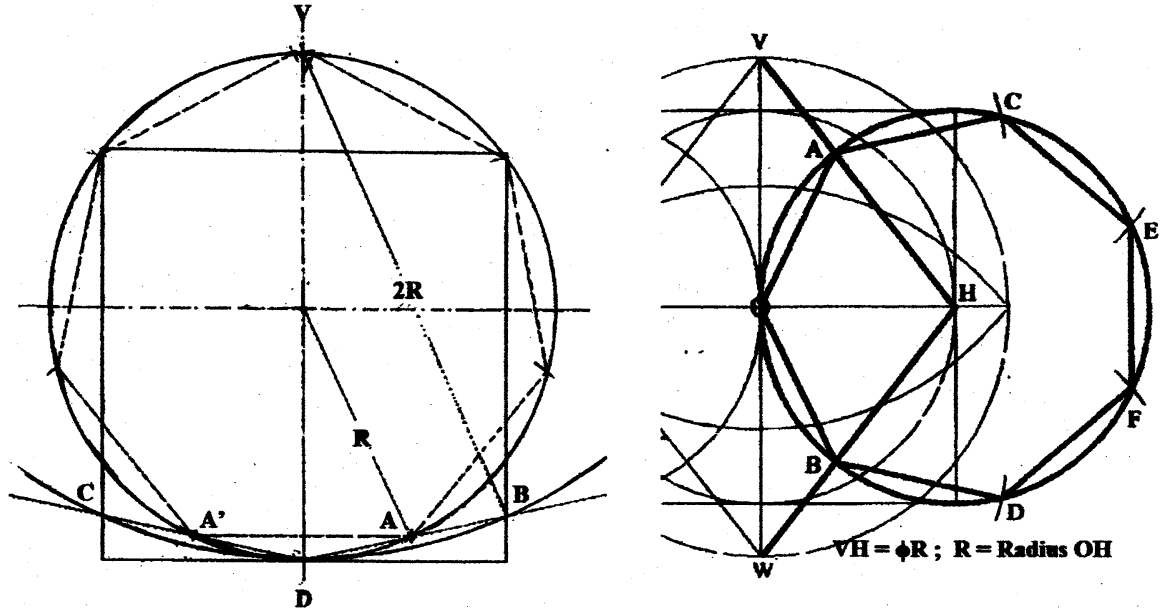

Fig. 5 (a, left) Arc/secant construction of the heptagon based on parameters side of square $\mathrm{L}=\phi \mathrm{R}$ and arc radius $\Psi=2 \mathrm{R}$ (i.e., 2R-Arc construction); (b, right) construction of the heptagon with $\alpha=\phi \mathrm{R}$, partially reproduced from Fletcher (2007; Fig. 25), with annotations added by the present author to facilitate the comparison

values can be related directly to $\phi$ through simple integer multipliers, it has to be concluded that it impossible to obtain an accuracy better than $2 \%$ with the $2 \mathrm{R}$-Arc construction of the heptagon based on $\phi$. Notwithstanding the lack of precision of this construction, geometer Rachel Fletcher (2007) has successfully devised a suitable procedure for the construction of the heptagon, using the golden ratio as a controlling parameter, which is identified as "square-the-circle". The construction is obtained by drawing two concentric circles with diameters defined by the diagonals of a rhombus exhibiting a ratio side $\div$ minor diagonal equal to $\phi$. Since the minor diagonal of the rhombus is twice the radius of the circumscribing circle, we can define a characterizing parameter $\alpha$ for this construction as the ratio of the side of the rhombus to the radius of the circle, shown in Fig. $5 \mathrm{~b}$ in comparison with the 2R-Arc construction with arc length $\mathrm{L}=\phi \mathrm{R}$. Rachel Fletcher claims an accuracy of $0.3 \%$ for the resulting heptagon.

A suitable procedure for the construction of a heptagon, through the use of the golden ratio $\phi$, has also been devised by Mark Reynolds (2001) as an evolution of the method used by Albrecht Dürer for the construction of the pentagon.

\section{Generalization of the 2R-Arc and Square-the-Circle Procedures}

The likely applicability of the $2 \mathrm{R}$-Arc procedure and the high accuracy of the "square the circle" method for the construction of the heptagon has provided the incentive to consider the two approaches as a means of devising generic procedures for the construction of polygons through the identification of the constitutive parameters. As controlling parameters to locate the vertices of a polygon, 
respectively the height of the chord, $\Psi-h$ (where $\Psi=$ Arc radius, h $=$ sagitta of the arc) was used for the 2R-Arc construction and the rhombus $\alpha$ ratio (defined earlier) for the Fletcher construction. The generalization of these two procedures is demonstrated by the examples shown in Fig. 6 for the construction of the pentagon, hexagon and octagon.

The Autocad work has shown that both methods produce exact constructions for the hexagons and octagon, while the constructions of the pentagon can be regarded as "highly precise": the 2R-Arc construction displays a deviation from the equilateral pentagon of less than $+0.001 \%$, while for the square-the-circle method
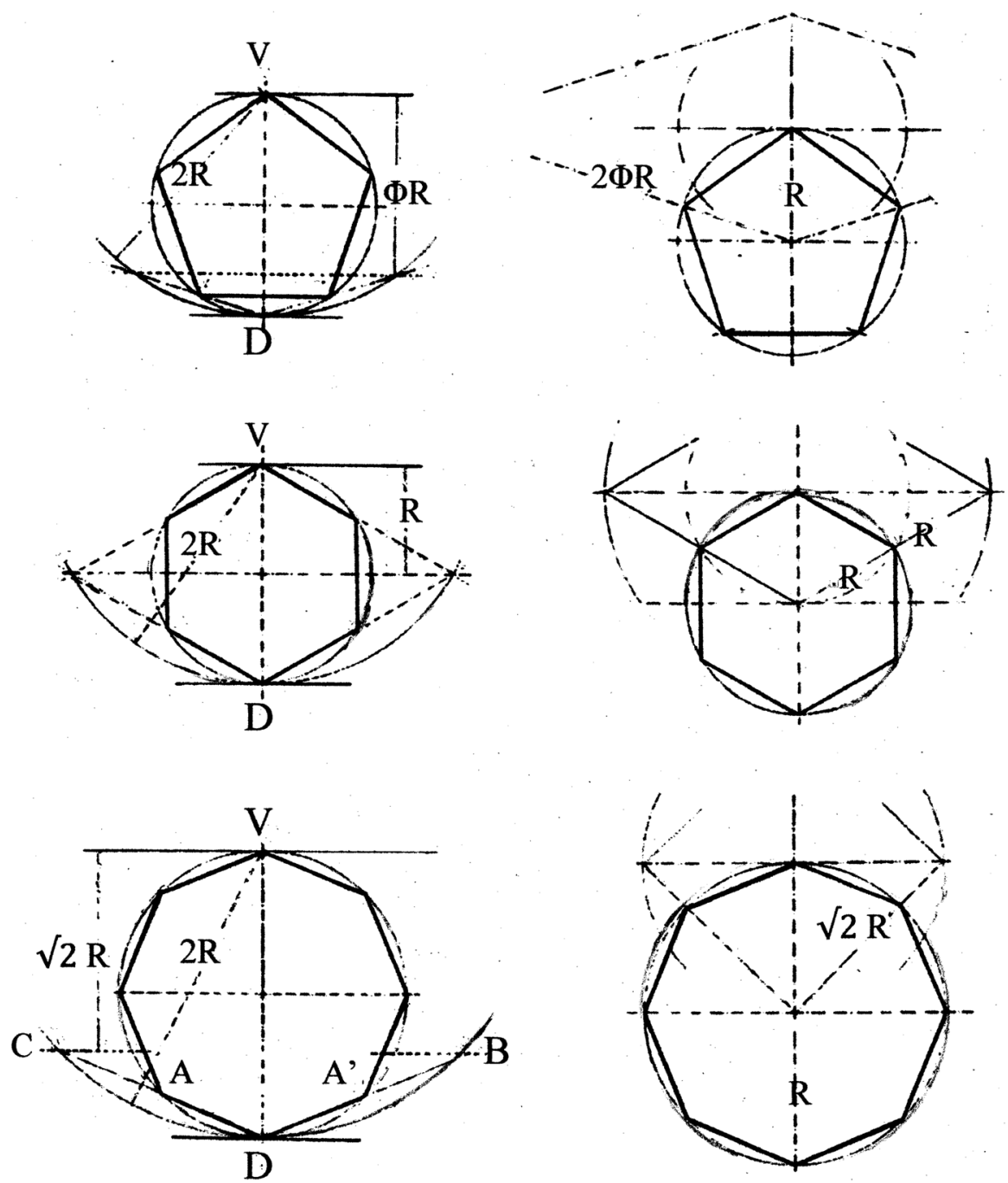

Fig. 6 Comparison of the 2R-Arc (left) with "the square the circle" (right) constructions of the pentagon, hexagon and octagon 
Table 1 Constitutive parameters for the construction of polygons by the 2R-Arc and Square-the-Circle methods

\begin{tabular}{llll}
\hline & Pentagon & Hexagon & Octagon \\
\hline 2R-Arc & & & \\
$\quad$ Chord height $(\Psi-\mathrm{h})$ & $\phi \mathrm{R}$ & $\mathrm{R}$ & $\mathrm{R} \sqrt{ } 2$ \\
$\begin{array}{l}\text { Square-the-circle } \\
\alpha \text { Ratio (side rhombus } \div \text { radius circle) }\end{array}$ & $2 \phi$ & 2 & $\sqrt{ } 2$ \\
\hline
\end{tabular}

the deviation is approximately $+0.002 \%$. The parameters used by the two constructions methods for the polygons in Fig. 6 are summarised in Table 1. Note that the connection between the controlling parameters for the pentagon $(\alpha=2 \phi)$ and for the heptagon $(\alpha=\phi)$ arises from the relationship between the vertex angles of the constitutive triangles delineated by the respective sides of the rhombus, i.e.,

$$
\frac{1}{2 \cos \left(\frac{2 \pi}{5}\right)}=\phi \approx \frac{1}{\cos \left(\frac{2 \pi}{7}\right)}
$$

As expected, the method was found to be applicable also for the construction of higher-order polygons, such as the decagon from the pentagon and dodecagon from the hexagon. However, attempts have failed to identify simple coefficients to obtain reasonably accurate procedures (i.e., deviations less than $1 \%$ ) for the construction of the nonagon and undecagon for both procedures.

\section{Peculiarities of Basic Polygons}

There is a certain controversy regarding the equilateral triangle and the square in the treatment of polygons. Although the equilateral triangle can be regarded as an elementary polygon, i.e., a polygon of lowest order, it has two features that do not satisfy the conditions applicable to all other polygons: (a) the sum of the internal angles is $180^{\circ}$ instead of $360^{\circ}$; (b) the triangle has no diagonals. The square, on the other hand, has a diagonal and satisfies the condition for the sum of internal angles being equal to $360^{\circ}$; however, it occupies a unique position in having the angle subtended by the sides equal to the central vertex angle of the constituent triangles.

In the light of the peculiarities inherent to the equilateral triangle and the square, the methods discussed earlier were examined in more detail to determine whether their construction fits with the two general procedures. The parameters that were identified for the two constructions are listed in Table 2 and the construction details are shown in Fig. 7. Autocad was used to verify that the both constructions provide exact solutions.

The details of the two constructions shown in Fig. 7 and the magnitude of the related parameters in Table 2 show that the $2 \mathrm{R}$-Arc procedure is applicable only for the construction of the equilateral triangle, which allows it to generate the corresponding hexagon through the secant method to generate the lower side vertices. The procedure for the square used by the $2 \mathrm{R}$-Arc, however, does not lead to the 
Table 2 Constitutive parameters for the construction of elementary polygons

\begin{tabular}{ll}
\hline 2R-Arc method & \\
\hline Chord height $(\Psi-\mathrm{h})$ & Related polygon and orientation \\
\hline $\mathrm{R}$ & $\begin{array}{l}\text { Equilateral triangle (horizontal base) } \\
\text { Square (vertical diagonal corners) }\end{array}$ \\
\hline 0 & \\
\hline Square-the-circle method & Related polygon and orientation \\
\hline Side rhombus/radius circle $(\alpha)$ & Equilateral triangle (inverted) \\
\hline$\sqrt{3}$ & Square (horizontal) \\
\hline$\sqrt{2}$ &
\end{tabular}
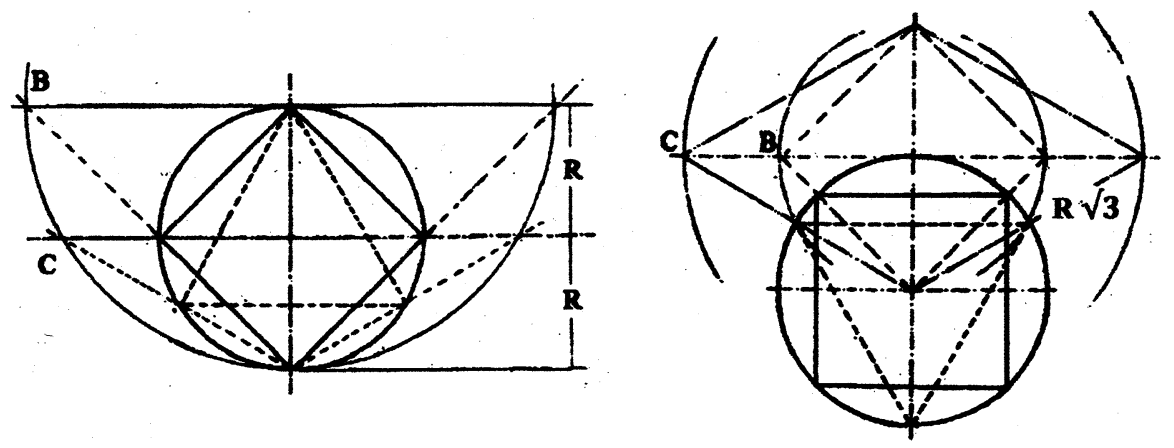

Fig. 7 Construction of the equilateral triangle and square. (a, left) Procedure "2R-Arc"; (b, right) Procedure "Square the circle"

evolution of the octagon, i.e., the corresponding higher-order polygons. The "square the circle" procedure displays the same dilemma but in the reverse order, insofar as the extrapolated parameter $\alpha$ only allows the square to evolve into a procedure for the higher-order polygon, i.e., the octagon. The inherent lack of generality of the procedures for the construction of the triangle and square has to be associated with the intrinsic anomalies identified earlier for these two elementary polygons.

\section{Conclusions}

The analysis of the Vitruvian man drawings by three Renaissance artists has revealed common features for these historical works that are rooted in geometry. It has emerged that the sketch nominally attributed to Giacomo Andrea da Ferrara and the one by Gulielmo Philandro, both delineate a hexagon as an evolution of the equilateral triangle with the apex at the centre of the circle. Due to the downward displacement of the equilateral triangle in the drawing by Leonardo da Vinci, which locates the vertex at the centre of the circumscribing circle, the polygonal inscription corresponds to that of a heptagon. 
Through experimentation on the use of arcs, both internal and tangential, suitable procedures have been identified for the direct construction of polygons of different orders up to the octagon. A general criterion was derived based on the geometric dimensions of a tangential arc with radius $(\Psi)$ twice that of the circumscribing circle $(\mathrm{R})$. The coefficients defining the ratio of diagonals in lower order polygons, such as $\sqrt{2}$ for the square and $\phi$ for the pentagon, were used to stipulate a suitable coefficient of $\mathrm{R}$ for each polygon. The least accurate construction was that used for the heptagon. The validity of the criterion was verified also for higher-order polygons by extrapolation, e.g., the decagon from the pentagon and the dodecagon from the hexagon.

The analysis has shown that the procedure used by geometer Rachel Fletcher for the construction of the heptagon also fits into a general criterion using parametric values for the ratio of the side to the minor diagonal of a rhombus as a means of locating the vertices of different polygons. Remarkably, the coefficients used to characterize the dimensions of the rhombus as the basis for the Fletcher construction were identical to those used for the dimensions of the tangential arc, which must be taken as evidence for the sound mathematical basis of both constructions.

Acknowledgments I wish to express my deepest gratitude to Daniele Battegazzore, studying for a Ph.D. in the Department of Applied Science and Technology at the Politecnico di Torino, for having devoted with enthusiasm a considerable amount of his free time to reproduce the various constructions of polygons discussed in the text, as a means of verifying the accuracy of each procedure. Without his help the publication of this work may have not been possible.

\section{References}

Agrippa, Henry Cornelius. 2003. Three Books of Occult Philosophy (1651). St. Paul: Lewellyn Publications.

Fernández, Margarita. 2005. Da Leonardo a Barbaro. Lettura grafica dell'uomo vitruviano.

From Leonardo to Barbaro. Graphic interpretation of the Vitruvian Man. Disegnare. Idee immagini 30: 10-19.

Fletcher, Rachel. 2007. Squaring the Circle: Marriage of Heaven and Earth. Nexus Network Journal 9: 119-144.

Helbing, Mario Otto. 2005. Il Poligono Reietto. Chiasso: Edizione Leggere.

Lester, Toby. 2012. Da Vinci's Ghost: Obsessions and How Leonardo Created the World in his Own Image. New York: Free Press.

March, Lionel. 1998. Architectonics of Humanism: Essays on Numbers in Architecture. London: Academy Editions.

Murtinho, Victor. 2015. Nexus Network Journal 17, 2. doi:10.1007/s0004-015-0247-7.

Reynolds, Emma. 2012. Da Vinci's iconic Vitruvian Man 'was copied from a fellow Renaissance architect'. Daily Mail Online, updated 08:28, 1 February 2012. http://www.dailymail.co.uk/news/ article-2094647/Leonardo-da-Vincis-Vitruvian-Man-copied-Giacomo-Andrea-da-Ferrara.html\#ixzz 2rUxGCOW2. Accessed 22/05/2015.

Reynolds, Mark A. 2001. From Pentagon to Heptagon: A Discovery on the Generation of the Regular Heptagon from the Equilateral Triangle and Pentagon. Nexus Network Journal 3, 2: 139-145.

Pedoe, Dan. 1976. Geometry and the Liberal Arts. London: Penguin Books.

Richter, J. P. 1970. The Notebooks of Leonardo da Vinci, 2 vols. (1883). New York: Dover Publications. Zöllner, Frank. 1985. Agrippa, Leonardo and the Codex Huygens. Journal of the Warburg and Courtauld Institutes 48: 229-234. 
Leno Liberato Mascia was born in Italy, where he attended schools up to the age of 19 before graduating in the UK, taking a first degree in Applied Chemistry, then a Ph.D. and received later the Doctor of Science award for his contribution to the Advancement of Science. He is now retired, having worked as a Research Scientist first at I.C.I. and then at Raychem Corporate Technology, holding also academic posts at Aston University and at Loughborough University. While he is still active in his main area of research, he has also developed an interest in Leonardo da Vinci and has initiated personal studies aimed to understand, and quantify, Leonardo's inclusion of science and mathematics concepts in art. 